\title{
Incidencia de las políticas de cooperación Sur-Sur sobre la orientación de la internacionalización universitaria en Argentina 2007-2015
}

SOLEDAD OREGIONI*

Artículo recibido: 20 de mayo de 2020

Artículo aprobado: 14 de abril de 2021

Doi: https://doi.org/10.12804/revistas.urosario.edu.co/desafios/a.8376

Para citar este artículo: Oregioni, S. (2021). Incidencia de las políticas de cooperación SurSur sobre la orientación de la internacionalización universitaria en Argentina 2007-2015. Desafíos, 33(2), 1-33. https://doi.org/10.12804/revistas.urosario.edu.co/desafios/a.8376

\section{Resumen}

A principios del XXI se observa la confluencia de dos fenómenos distintos, pero directamente vinculados, en la orientación de la politica de internacionalización de las universidades argentinas. Por un lado, el avance del proceso de internacionalización de la Educación Superior en América Latina y, por otro, el giro de la politica regional basada en priorizar los lazos de cooperación Sur-Sur (CSS). El artículo describe y analiza cómo las políticas de CSS en ciencia, tecnología y universidad inciden en el proceso de internacionalización universitaria. Con base en las politicas de CSS impulsadas por el Estado Nacional, en el periodo 2007-2015, a través de la Secretaría de Políticas Universitarias del Ministerio de Educación; el Ministerio de Ciencia, Tecnología e Innovación Productiva; y el Fondo Argentino de Cooperación Sur-Sur

\footnotetext{
* CONICET-UNICEN-CEIPIL/CIC. Correo electrónico: soregioni08@hotmail.com. ORCID: http://orcid.org/0000-0002-5741-0428
} 


\title{
2 I Soledad Oregioni
}

y Triangular (FOAR), dependiente de la Dirección de Relaciones Internacionales del Ministerio de Relaciones Exteriores, Comercio Internacional y Culto. Esto da lugar a discutir la relación agencia-estructura, en relación al proceso de internacionalización universitaria.

Palabras clave: cooperación sur-sur; internacionalización universitaria; cooperación en ciencia y tecnología; Argentina.

\section{Impact of South-South Cooperation Policies on the Orientation of University Internationalization in Argentina 2007-2015}

\begin{abstract}
At the beginning of the XXI century, the confluence of two different, but directly linked, phenomena is observed, in the orientation of internationalization policies in Argentine universities. On the one hand, the progress of the internationalization process of Higher Education in Latin America and, on the other, the shift in regional policy based on prioritizing South-South cooperation ties (SSC). The article aims to describe and analyze how CSS policies in science, technology and university affect internationalization. I focus on the CSS policies promoted by the National State in the period 2007-2015 through: the Secretariat for University Policies of the Ministry of Education; the Ministry of Science, Technology and Productive Innovation; and the Argentine Fund for South-South and Triangular Cooperation (FOAR), dependent on the Directorate of International Relations of the Ministry of Foreign Affairs, International Trade and Worship. In this context I discuss the agency-structure relationship in relation to the university internationalization process.
\end{abstract} Keywords: South-south cooperation; university internationalization; cooperation in science and technology; Argentina. 


\section{Impacto das políticas de cooperação Sul- Sul na orientação da internacionalização universitária na Argentina 2007-2015}

\section{Resumo}

No início do século XXI, observa-se a confluência de dois fenômenos distintos, mas diretamente ligados na orientação da política de internacionalização das universidades argentinas. De um lado, o avanço do processo de internacionalização da Educação Superior na América Latina e, de outro, a mudança da política regional baseada na priorização dos laços de cooperação Sul-Sul (CSS). O artigo tem o objetivo de descrever e analisar como as politicas de CSS em ciência, tecnologia e universidades afetam oprocesso de internacionalização universitária. Com base nas politicas de CSS promovidas pelo Estado Nacional no período 2007-2015, por meio: da Secretaria de Políticas Universitárias do Ministério da Educação; Ministério da Ciência, Tecnologia e Inovação Produtiva; e o Fundo Argentino de Cooperação Sul-Sul e Triangular (FOAR), dependente da Diretoria de Relacõos Internacionais do Ministério das Relações Exteriores, Comércio Internacional e Culto. Este fato dá lugar à discussão da relação agência-estrutura, no que se refere ao processo de internacionalização universitária. Palavras-chave: cooperação sul-sul; internacionalização universitária; cooperação em ciência e tecnologia; Argentina.

\section{Introducción}

En los inicios del siglo XXI se observa la confluencia de dos fenómenos distintos, pero directamente vinculados a tensionar la orientación de la internacionalización las universidades en Argentina. Por un lado, el avance del proceso de internacionalización de la Educación Superior en América Latina y, por otro lado, el giro en la política regional, a partir de priorizar lazos de Cooperación Sur-Sur (CSS).

En cuanto al primero, desde una perspectiva histórica y contextual, podemos observar que las universidades son internacionales desde sus orígenes y el desarrollo de la ciencia moderna históricamente se ha retroalimentado, a partir de la vinculación internacional de los científicos, sin embargo, desde el último cuarto del siglo $\mathrm{xx}$, 
a raíz de cambios de orden cuantitativos y cualitativos, respecto a las dinámicas de vinculación internacional, se comienza a hacer referencia a la internacionalización como proceso (Altbach, 2004; García-Guadilla, 2010), visibilizando distintas lógicas que pugnan por su orientación, que en trabajos previos se identificaron como internacionalización hegemónica, no-hegemónica y contra-hegemónica (Oregioni, 2017; Oregioni \& Piñero, 2017; Leal \& Oregioni, 2019).

Consecuentemente, el proceso de internacionalización universitaria se caracteriza por ser multidimensional, complejo y no neutral. Se encuentra orientado por organismos internacionales, regionales, nacionales, institucionales y sectoriales, a partir de políticas de promoción (incidencia directa) y de evaluación ${ }^{1}$ (incidencia indirecta) (Oregioni, 2016). En este sentido, las políticas de cooperación en ciencia, tecnología y universidad, consisten en una de las aristas de análisis (aunque no la única) a tener en cuenta para comprender su orientación, debido a que constituyen un estímulo directo para la internacionalización de las universidades.

En cuanto al giro político hacia la integración regional, que se evidencia a inicios del siglo XXI, expresa la búsqueda de una inserción internacional más autónoma que reivindica la cooperación Sur-Sur (Serbin et al., 2012) y desafía los patrones tradicionales de comercio y poder hegemónico, que históricamente se identificaron con el poder desproporcionado de Estados Unidos en la Región. De esta forma, el regionalismo posliberal (Sanahuja, 2010) o poshegemónico (Riggirozzi \& Tussie, 2012), implica pensar la región como un espacio de discusión y acción colectiva, que excede la dimensión comercial (Grugel \& Riggirozzi, 2012) y le da mayor protagonismo a la dimensión

\footnotetext{
1 Los parámetros de evaluación ejercen una incidencia indirecta en el proceso de internacionalización universitaria, a partir de los criterios utilizados para evaluar a los investigadores y a las instituciones de educación superior. Estos son ponderados a partir de rankings internacionales, que establecen criterios de calidad y excelencia. En ese sentido, los rankings especializados de Estados Unidos, Europa y Asia se han constituido en modelos normativos para las instituciones de Educación Superior de América Latina, en el marco de una internacionalización competitiva (Oregioni, 2016).
} 
educativa. Esto se evidencia en la dinámica que tuvo, por ejemplo, el Sector Educativo del Mercosur.

En el caso de Argentina, desde el año 2003 con la elección de Néstor Kirchner como presidente de la Nación, se rompió con los preceptos neoliberales y se redefinió el rol del Estado como impulsor del desarrollo con inclusión social. Política que tuvo continuidad durante el mandato de Cristina Fernández (2007-2015), quién le otorgó a la región latinoamericana, en general, y suramericana, en particular, un rol privilegiado en su política exterior (Fernández, 2019).

El artículo presenta el objetivo de describir y analizar cómo inciden las políticas de cooperación Sur-Sur en ciencia, tecnología y universidad, en el proceso de internacionalización universitaria. A partir de puntualizar en las políticas de css que promueve el Estado Nacional, a través de la Secretaría de Políticas Universitarias, dependiente del Ministerio de Educación; del Ministerio de Ciencia, Tecnología e Innovación Productiva; del Fondo Argentino de Cooperación SurSur y Triangular (FOAR), dependiente de la Dirección de Relaciones Internacionales del Ministerio de Relaciones Exteriores, Comercio Internacional y Culto. Es decir: ¿Las políticas de CSS, promovidas desde dichas dependencias nacionales, generaron instrumentos que permitan orientar el proceso de internacionalización universitaria hacia la Región, en el contexto de Regionalismo posliberal o poshegemónico?

Particularmente, se indagó en el carácter situado de la internacionalización, a partir de puntualizar en las políticas de css que implementó Argentina en el periodo 2007-2015. En primer lugar, porque se correlaciona con la orientación explicita de la política exterior y, en segundo lugar, por la relevancia que adquieren las políticas de cooperación SurSur en la orientación de dinámicas de producción de conocimiento, a partir de agendas endógenas a la región latinoamericana.

En este sentido, de acuerdo con la perspectiva teórica de Boaventura de Sousa Santos, la universidad, en vinculación con otros actores sociales como el Estado, ocupa un lugar central en la construcción 


\section{6 / Soledad Oregioni}

de alternativas contra hegemónicas a la globalización neoliberal, a partir de orientar el proceso de internacionalización mediante políticas de cooperación, que permitan la construcción de conocimiento relevante, en interacción con otros actores sociales y tipos de saberes (De Sousa-Santos, 2008).

Se trabaja con la hipótesis que Argentina buscó tejer alianzas estratégicas en función de un proyecto nacional y regional, que entró en tensión con elementos estructurales e incentivos globales. De esta forma, el Estado argentino generó políticas tendientes a orientar la internacionalización de las universidades mediante instrumentos de CSS, que contribuyeron a tensionar el proceso de internacionalización hegemónico. $^{2}$

El artículo se organiza en cuatro secciones. En la primera, se presenta un recorrido conceptual que permitirá comprender la relevancia de la Css en el proceso de internacionalización universitaria. En segundo lugar, se identificó a las políticas de cooperación, como un elemento que incide en forma directa en la orientación de la internacionalización, particularmente, se puntualizó en las políticas de CSS orientadas hacia la Región, que implementó Argentina en el periodo 2007-2015, acorde a los objetivos de su política exterior. En tercer término, se analiza la relación agencia-estructura en la co-construcción de procesos sociales, como es el proceso de internacionalización, desde una perspectiva situada en la Región latinoamericana. Por último, a modo de conclusión, se presentan las reflexiones que emergen de la investigación.

\section{Problemas de la Cooperación Norte-Sur y relevancia de la Cooperación Sur-Sur en la internacionalización universitaria}

Los Estados alimentan la proyección internacional de la universidad y de sus investigadores a partir de su política exterior y de su política

2 Ver: Oregioni, 2015. 
científica, que confluyen en la política de cooperación en ciencia y tecnología (Kern, 2008). Asimismo, esta relación se complejiza cuando incorporamos al análisis a las comunidades productoras de conocimiento, que se desempeñan en las universidades, desde una perspectiva situada en la Región Latinoamericana. De este modo, para comprender qué se pone en juego con la internacionalización de las universidades, es preciso reparar en su carácter multidimensional, contemplando los aspectos socio-cognitivos y político-institucionales. Los primeros, permiten entender cómo incide la internacionalización en las dinámicas de producción de conocimiento, mientras que los segundos, dan cuenta de los instrumentos de política que contribuyen a su orientación (Oregioni, 2014).

Desde los estudios sociales de la ciencia, se ha avanzado en analizar la internacionalización en el ámbito del laboratorio, contemplando las asimetrías de poder que sustentan y reproducen un sistema cognitivo internacional inequitativo y jerárquico. En este sentido, los aportes de Pablo Kreimer (2014) hacen referencia a los distintos momentos de la internacionalización de la investigación en América Latina y su contribución en la construcción de campos científicos, observando que, en los albores del siglo xxI, la negociación de los problemas cognitivos a trabajar se tornó más dependiente de los centros de producción de conocimiento que elaboran contratos cerrados, de tipo "tómalo o déjalo", en un contexto geopolítico, donde las potencias hegemónicas han ingresado en una competencia global por el desarrollo de capacidades, para la innovación e investigación científica, en el marco de una estrategia competitiva mucho más amplia. Donde históricamente competían Estados Unidos y Europa, actualmente se incorporan otros actores no occidentales, como es el caso de China, planteando nuevos desafíos.

Siguiendo a Kreimer (2014), lo anteriormente expuesto impacta en la integración subordinada que ha sufrido históricamente la investigación latinoamericana, a partir de: 1) restricción del margen de negociación por parte de los grupos periféricos; 2) proceso de división internacional del trabajo científico, que asigna a los investigadores 


\section{8 / Soledad Oregioni}

latinoamericanos un lugar fuertemente especializado, con alto contenido técnico, pero con poca incidencia en la definición de los problemas cognitivos a trabajar; 3) reproducción ampliada que permitirá incorporar nuevos métodos y técnicas, que luego utilizarán al retomar a sus países. Así, "este nuevo tipo de internacionalización deja poco margen para reformular problemas sociales y locales como problemas del saber" (Kreimer, 2014, p. 158), ya que las asimetrías dificultan la posibilidad de negociar los problemas cognitivos a trabajar y llevan a direccionar las agendas de investigación exógenamente.

De esta forma, la "internacionalización hegemónica" de carácter exógeno, se manifiesta a partir del incentivo directo de proyectos, agentes, actores e intereses extra regionales, que históricamente guiaron las políticas de cooperación norte-sur (Oregioni, 2017), de acuerdo a los ideales de la "modernidad occidental" y el "desarrollo lineal", entendiendo que los pueblos deben pasar desde lo primitivo a lo tradicional, luego, a lo moderno, y la sociedad liberal europea es el punto de llegada (Dussel, 2000). Ahora bien, ¿a quienes beneficia y a quienes perjudican dichas relaciones?

Los estudios sobre dependencia académica e intelectual, desde la década del sesenta han reunido a importantes investigadores de África, Asia y América Latina quienes cuestionan el colonialismo y sus efectos (Pinhero \& Martin, 2014). De acuerdo con Beigel \& Sabea, "el concepto de dependencia académica se refiere a la estructura desigual de producción y difusión del conocimiento construida históricamente en lo que conocemos como sistema científico internacional" (2014, p. 15), sin embargo, Beigel se aleja de una mirada lineal y simplista del fenómeno e identifica el concepto de "heterogeneidad estructural", que refiere a la complejidad de estas asimetrías, dado que el campo se encuentra atravesado por circuitos transnacionales, regionales, y nacionales (Beigel, 2013). En la misma línea, De Sousa Santos plantea la incidencia de las relaciones de poder en las dinámicas de producción de conocimiento, y la relación poder/saber, afirmando que hasta ahora ese conocimiento científico estuvo en los países del norte, deslegitimando otro tipo de saberes (De Sousa-Santos, 2017). 
De acuerdo a los antecedentes provenientes de los estudios sociales en ciencia y tecnología, la cooperación históricamente se orientó desde los países del Norte (Cetto \& Vessuri, 1998). Mientras que, a pesar de la cercanía, en términos históricos y culturales, entre los países latinoamericanos, predominaron los lazos mediados por Estados Unidos y Europa (Marí et al., 2001). Los estudios bibliométricos, que toman a las publicaciones como el principal output del proceso de producción de conocimiento, para analizar la interacción entre investigadores latinoamericanos, ${ }^{3}$ dan cuenta de esta tendencia (Fernández et al., 1998; Sancho et al., 2006; Russell et al., 2007; entre otros).

La principal crítica a las dinámicas de cooperación norte-sur, consiste en que generalmente llevan a investigar problemas que no responden al contexto social, político y económico donde se desarrolla la investigación, ya que los países "periféricos" integran redes muy amplias, cuyos programas ya han sido sólidamente estructurados por quienes los financian, consecuentemente, en la división del trabajo científico los investigadores de la periferia tienen un rol sumamente técnico (Kreimer \& Levin, 2011). De esta forma, se considera necesario trabajar en relación a la legitimidad y pertinencia de estas prácticas (Oregioni \& Piñero, 2009).

Teniendo en cuenta lo planteado en los párrafos precedentes, a continuación, se analizará la relevancia que adquieren los aspectos políticoinstitucionales, al promover políticas de css en relación a la proyección política del Estado Argentino, en el periodo 2007-2015, donde primaban ideas posneoliberales, vinculadas al regionalismo poshegemónico.

\section{Política de cooperación internacional en Ciencia y Tecnología}

Tal como se ha mencionado en trabajos previos, si bien las actividades de vinculación entre investigadores han sido centrales en la

\footnotetext{
3 Ver la critica que se realiza a la efectividad de los indicadores bibliométricos para medir la internacionalización de la investigación en Oregioni (2014).
} 
construcción de trayectorias científicas en los países latinoamericanos, la política de cooperación en ciencia y tecnología, como política pública, emerge a partir de la segunda mitad del siglo xx (Oregioni \& Piñero, 2009).

Ahora bien, entender la politica de cooperación internacional en ciencia, tecnología como política pública implica pensarla en el contexto de un proyecto nacional, es decir, del conjunto de objetivos al que aspiran los sectores sociales, que ejercen el control político y económico de un país (Oregioni \& Abba, 2012). En este sentido, de acuerdo con Hurtado (2012), se sostiene la importancia de articular la política de cooperación en ciencia y tecnología con la política nacional de desarrollo, entendiendo que no toda cooperación es buena en sí misma, sino que exige establecer criterios en su orientación.

Así, uno de los principales debates que han surgido en torno a las políticas de cooperación en ciencia y tecnología, se relaciona con su orientación. Si bien, en los países latinoamericanos, los tomadores de decisiones tienden a considerar que "toda cooperación es bienvenida", con base en una concepción universalista de la ciencia, desde el análisis académico se ha contribuido a cuestionar su carácter neutral y a revalorizar los vínculos de CSS, particularmente hacia el ámbito regional (Velho, 2000). Entendiendo que las respuestas a los problemas regionales deben proyectarse en función de agendas endógenas, debatidas y consensuadas por las sociedades latinoamericanas. Para esto, es imperante descolonizar el pensamiento y el conocimiento para que el Sur Global sea un proyecto viable que genere cambios reales.

En la bibliografía, existe dispersión conceptual en torno a la CSS, ya que no solo se refiere a una interpretación espacial o geográfica, sino que pone en juego las reivindicaciones, alianzas y dinámicas históricas de los países más desfavorecidos de sistema internacional. Consecuentemente, las dimensiones de análisis e indicadores trabajados para comprender CSS en ciencia y tecnología, no dan cuenta de la dinámica que adquieren estas prácticas en los países latinoamericanos. 
En el campo de estudios sobre Relaciones Internacionales, se identificaron dos tendencias en la definición de CSS, una que se promueve desde los organismos multilaterales y otra que tiene en cuenta los postulados de la política exterior (Colacrai et al., 2009).

En el primer caso, desde los inicios del siglo XXI, se observan continuidades y cambios en la definición de CSs. Respecto a las continuidades, subsiste la idea que mejora la inserción de los países del Sur en el contexto económico internacional, caracterizado por la globalización de los mercados y la producción, y no como una instancia superadora de un orden internacional injusto (como se la concebía en los años 70). En cuanto a los cambios, se identificó que se ha optado por el concepto de CSS, ampliando la denominación de cooperación entre países en desarrollo.

Por ejemplo, en el ámbito de Naciones Unidas se han incorporado la cooperación triangular y, en las declaraciones multilaterales, se tiende a identificar la CSS como un aspecto complementario de la cooperación Norte-Sur. De esta forma, la nueva tendencia de la CSS, implicaría que se benefician tanto receptores como donantes de la cooperación, a partir del incremento de sus capacidades, ya que al colaborar se vinculan recursos provenientes de cooperación internacional con las propias necesidades nacionales. Así la cooperación triangular generaría una nueva modalidad de vinculación entre cooperantes, al articular las tradicionales relaciones de cooperación Norte-Sur con la cooperación entre países del Sur, e identificar posibles sinergias que contribuyan a la construcción de un sistema internacional más justo (Alonso et al., 2011).

En el segundo caso, se toma en cuenta la orientación de la política exterior. De esta forma, se entiende a la CSS como una estrategia interna de orientación de la política exterior de un país. Por ejemplo, Collin (2001) relaciona la cooperación académica y científica como un aspecto de la política exterior interna de cada país y con su perfil en la escena internacional. En esta misma línea, Surasky (2010) sostiene que la cooperación internacional que realiza Argentina se encuentra directamente ligada a los objetivos de política exterior, consecuentemente, se 
ve afectada por los vaivenes de la política nacional. En tanto, Malacalza (2014) afirma que, si bien no siempre es posible identificar un tronco común para caracterizar a la literatura latinoamericana sobre CSS, esta se distingue por desarrollar experiencias concretas, con base en estudios de caso, ${ }^{4}$ dando cuenta de que refleja dinámicas, ideas e intereses diferentes a los de las dinámicas de cooperación Norte-Sur.

En el presente trabajo, se identifica la política de Css en ciencia y tecnología con la orientación de la política exterior. En este sentido, se contemplan las tensiones entre "dependentistas" y "autonomistas" en el diseño de la política exterior (Simonoff, 2009) y se las relaciona con las estrategias endógenas y exógenas de internacionalización universitaria, a partir de la promoción de políticas de cooperación en ciencia y tecnología.

Esto quiere decir que cuando se hace referencia a la cooperación internacional, como una estrategia de política exterior, es necesario pensarla más allá de la cancillería, dado que forma parte de una política pública que se desarrolla desde diferentes dependencias del estado, en coherencia o no con un proyecto nacional. Ahora bien, ¿hacia dónde se orienta la política exterior argentina en el periodo de estudio? ¿Qué instrumentos de CSS contribuyen a dicha orientación? ¿Cómo incide esto sobre el proceso de internacionalización universitaria?

De acuerdo con Alejandro Simonoff (2009), desde 1983 la política exterior argentina se construyó a partir de un juego de equilibrios entre "autonomistas" $\mathrm{y}$ "dependentistas", que se distingue a partir

\footnotetext{
4 En este sentido, el autor identificó distintos trabajos como los de Hirst \& Soares de Lima, 2006; Lechini, 2009; Lechini \& Giaccaglia 2010; Hirst, 2010; 2011, 2012; Kern \& Weisstaub, 2010; Kern \& Rodrigues Patrinos, 2010; Ayllón \& Surasky, 2010; Lengyel \& Malacalza, 2011; 2012; Ayllón \& Ojeda, 2013. (Véase Malacalza, 2014)

5 De acuerdo con Morasso (2016), el concepto "autonomía” conservó el sentido que le asignó Puig en la década del ochenta, quien la define en términos de la capacidad de decisiones que puede lograr un Estado, teniendo en cuenta los condicionantes externos, esto implica que el Estado cuente con un modelo de desarrollo económico propio, es decir, que participe en el sistema internacional en función de sus propios intereses (Pereyra-Doval, 2014). En este sentido, es necesario que el grupo que ejerce el poder se identifique a sí mismo como
} 
del marco de alianzas estratégicas que están dispuestos a tejer y los objetivos que se plantean. Mientras que los "autonomistas" apuntan a generar márgenes de maniobras en el sistema internacional, sobre alianzas con países de similares recursos y valores, para poder llevar a cabo sus proyectos económicos internos, que buscan rever su posición en el sistema internacional. Los "dependentistas" han optado por una política de seguimiento a la potencia hegemónica, con el fin de estrechar aún más las relaciones derivadas de la actual división internacional del trabajo. ${ }^{6}$

En la década del noventa, en el contexto de una política exterior dependentista, se planteaba que la globalización traía aparejado el abandono de todo tipo de política, suponiendo que el Estado dejaría las decisiones libradas en manos del mercado. Sin embargo, en el periodo 20032015, el Estado manifestó la voluntad de retomar la conducción. De esta forma, los gobiernos nacionales se identifican como agentes de cooperación internacional ya que presentaban el objetivo de fomentar y articular la cooperación internacional de acuerdo con los intereses y la lógica de su política exterior, acorde a ciertas prioridades temáticas y geográficas, particularmente, hacia el ámbito regional a partir de la generación de tratados de cooperación (Losego \& Arvanitis, 2008).

En la búsqueda de una política exterior autonomista, se destacan cambios radicales respecto a los vínculos con los Organismos Internacionales de crédito, principalmente el Fondo Monetario Internacional (FMI)

relativamente independiente del centro de poder internacional, con capacidad de trazar sus propios objetivos (Romero, 2009).

6 Por ejemplo, en los noventa, la política exterior argentina estuvo orientada por ideas neoliberales y dependentistas, así los objetivos de la política exterior se orientaron a la seducción a la potencia hegemónica, mediante una subordinación plena de intereses, y la aplicación de recetas propuestas por los Organismos Multilaterales de Crédito. En el plano regional se priorizaron cuestiones relacionadas a lo comercial, reduciéndose la vinculación con los países latinoamericanos, exclusivamente, a lo económico. Durante estos años la internacionalización de las universidades nacionales estuvo orientada de manera exógena debido a la importante influencia que ejercían los organismos internacionales, entre ellos el fmi, el bm y la Organización para la Cooperación y el Desarrollo Económico (ocde), entre otros (Oregioni y Daddario, 2016) 
como fue la cancelación de la deuda por un monto de 9.810 millones de dólares, utilizando el $36 \%$ de las reservas (Redrado, 2010). Esto se identifica como un símbolo de ruptura con el modelo neoliberal, característico de la década anterior, dependiente de este tipo de Organismos y sujeto constantemente a la aplicación de recetas y condicionamientos económicos.

En este sentido, el giro conceptual que da la política exterior argentina se relaciona con la decisión de no renunciar a la autonomía de las decisiones y participar de una manera activa en la construcción de un Nuevo Orden Mundial (Colombo, 2011). En el año 2005 esto se manifiesta con el "No al ALCA", que puso fin al intento norteamericano de crear un área de libre comercio en el continente, manifestando el compromiso de Argentina con la integración de América del Sur, que se formalizaría en el año 2008, a través del Tratado Constitutivo la Unión de Naciones Suramericanas y que entraría en vigor en marzo del 2011, luego de la correspondiente ratificación de cada una de las doce Naciones de América del Sur.

Asimismo, al no existir una normativa que institucionalice y regule las actividades de css dentro de un sistema integrado, se pueden presentar tensiones entre los objetivos institucionales y los objetivos de política exterior (Kern \& Weisstaub, 2019). Consecuentemente, surge como interrogante si las características de la política exterior argentina, en el periodo 2007-2015, se reflejan en la promoción de CSS en ciencia y tecnología, desde distintos organismos centralizados que tienen llegada a las universidades, generando elementos para orientar el proceso de internacionalización hacia la Región. Indagamos en las políticas de CSS que se promovieron desde Fondo Argentino de Cooperación Sur-Sur y Triangular (FOAR), desde el Ministerio de Educación, mediante la Secretaría de Políticas Universitarias (SPU) y desde el Ministerio de Ciencia, Tecnología e Innovación (MINCYT). ${ }^{7}$

\footnotetext{
En este último caso, si bien lleva adelante programas de css a partir de los cuales se han desarrollado importantes aprendizajes institucionales, en el periodo de estudio, continuó priorizando las dinámicas tradicionales de cooperación norte sur.
} 


\section{El Fondo Argentino de Cooperación Sur-Sur y Triangular (FOAR)}

La Dirección General de Cooperación Internacional (DGCIN) dependiente de la Secretaría de Coordinación y Cooperación Internacional del Ministerio de Relaciones Exteriores, Comercio Internacional y Culto (MRECIC) es la encargada de establecer los lineamientos de política exterior en materia de cooperación internacional. Sus objetivos estratégicos priorizan la promoción de la cooperación al desarrollo, el fortalecimiento del multilateralismo y a la profundización de la integración regional. En tanto, sus funciones se desempeñan mediante la cooperación bilateral y multilateral. Además, coordina la Sección Nacional del Comité de Cooperación Técnica (ССT) del Grupo Mercado Común del Mercosur. Ahora bien, la principal herramienta de Cooperación Técnica al Desarrollo de la DGCIN es el Fondo Argentino de Cooperación Sur-Sur y Triangular (FOAR), que se creó en el año 1992, como un instrumento para financiar la cooperación Sur-Sur, con el objetivo de asociarse con otros países a fin de generar estrategias de desarrollo con base en las prioridades nacionales.

A través del FOAR se llevan adelante estrategias de CSS, donde intervienen las universidades y sus investigadores. El organismo trabaja a partir de objetivos estratégicos, en relación al proyecto de política exterior nacional, en coordinación con otros actores, con el objetivo de apoyar la agenda de internacionalización de cada institución pública de la Argentina. Aunque cada Ministerio tiene su propia agenda de política internacional, se trata de coordinar aquellos objetivos que son de la Argentina en general y no de una institución en particular. Por ejemplo, la disminución de asimetrías en el Mercosur, que es un objetivo de política exterior de la Argentina en el periodo de estudio.

Desde el ámbito del FOAR, la cancillería argentina plantea la importancia de generar proyectos de cooperación a largo plazo. A partir del año 2003 se comenzó a trabajar en cuestiones estratégicas, se definieron: carteras, áreas, proyectos a dos años de ejecución, con planificación de actividades que contemplaban resultados o productos completos. De esta forma, se propuso como objetivo estratégico la cooperación con Bolivia y Paraguay con el fin de disminuir las asimetrías en la Región, 
de acuerdo a una política de Estado que priorizaba la Cooperación Regional (Oregioni \& Abba, 2012).

De acuerdo con Kern \& Weusstaub:

La Argentina ha sabido construir una marca propia a través del FOAR. Gracias a este instrumento ha podido ubicarse entre los principales oferentes de CSS de la región (SEGIB, 2016). Los distintos gobiernos, lo han sabido asimilar a sus estrategias políticas dándole continuidad en el marco objetivos de política exterior diversos. (2019, p. 42)

Dentro de las estrategias de cooperación internacional que se priorizaron, en el periodo 2007-2015, se identificó la vinculación con las universidades en proyección regional. Las actividades de cooperación consistían en el envío al exterior de expertos, provenientes de distintos Organismos de Ciencia y Tecnología y de las universidades, con el objetivo de dar respuesta a problemas regionales.

Por ejemplo, la Universidad Nacional de La Plata (UNLP) participó activamente de un proyecto, donde expertos de la Facultad de Ciencias Veterinarias capacitan, en genética molecular de bovinos, a profesionales del Laboratorio de ADN de la Facultad de Ciencias Veterinarias, de la Universidad Autónoma Gabriel René Moreno de Santa Cruz de la Sierra, Bolivia. Con ello se buscaba contribuir a implementar métodos de diagnóstico molecular y difundirlos, tanto en el ámbito académico como en el medio productivo.

En la proyección regional del FOAR los principales socios receptores para el periodo 2008-2015, en términos cuantitativos, han sido: Paraguay, con 420 proyectos; Bolivia, con 211 proyectos; el Salvador, con 110 proyectos; y Cuba, con 101 proyectos (Kern \& Weusstaub, 2019).

\section{Secretaria de Políticas Universitarias. Ministerio de Educación}

A partir del año 2003, el alineamiento entre la política exterior y política de CSs, llevada adelante por los gobiernos de Néstor Kirchner y Cristina 
Fernández, contribuyeron a orientar el proceso de internacionalización universitaria. Esto se puede observar a partir de acciones concretas emprendidas desde el Ministerio de Educación, mediante la Secretaría de Políticas Universitarias (SPU). Entre ellas se destacan las siguientes:

En primer lugar, la participación y el posicionamiento de Argentina en la Conferencia Regional en Educación Superior (CRES) del año 2008 (Conferencia Regional, 2009), con el objetivo de articular una posición común frente a los postulados globales y la búsqueda de una posición autónoma, comprendiendo la no neutralidad de la internacionalización de la educación superior. En este sentido, la representación argentina junto al resto de las delegaciones latinoamericanas, lograron consensuar un posicionamiento común frente al avance de la mercantilización de la educación superior. Consecuentemente, la región latinoamericana se posicionó a nivel global en defensa de la educación superior como un bien público y responsabilidad del Estado, en contraposición a las estrategias promovidas por los organismos financieros internacionales, tendientes a la mercantilización de la educación superior entendida como un "servicio" (Oregioni, 2013).

En segundo lugar, se identificaron distintos programas promovidos desde la SPU, que ponen el eje en el fomento de la CSS, como: "Consensos del Sur para el desarrollo con Inclusión Social"; los "Programas Redes", promovidos desde el Programa de Promoción de la Universidad Argentina (PPUA) en el periodo 2006-2015; los programas del español como segunda lengua; entre otros. ${ }^{8}$

Además, la SPU presentaba tres líneas de acción permanentes vinculadas a internacionalización de la educación superior, estas son:

\footnotetext{
8 Las distintas convocatorias del Programa de Promoción de la Universidad Argentina (PPUA) se desarrollaron sobre los ejes: a) Fortalecimiento de las Áreas de Relaciones Internacionales; b) Fortalecimiento de Redes Interuniversitarias; c) Misiones Universitarias al Extranjero; d) Puentes entre la Universidad y la Sociedad; e) Responsabilidad Social Universitaria; f) Fortalecimiento Institucional en Instituciones Universitarias; g) Fortalecimiento de la función de extensión; h) Actividades de perfeccionamiento en el exterior y participación en eventos internacionales; i) Fortalecimiento de la capacidad de gestión del área de vinculación tecnológica.
} 
1) la convocatoria a "Redes Académicas Interinstitucionales", donde se busca que las Universidades argentinas conformen o fortalezcan redes académicas con Universidades del exterior;

2) "Misiones al Extranjero", que consiste en financiar misiones al exterior para atraer estudiantes y socios internacionales;

3) Financiamiento de la promoción de la Universidad argentina en conferencias y ferias internacionales de Educación Superior, que se realiza conjuntamente con Cancillería y la Fundación Exportar.

En tercer lugar, el Programa de Internacionalización de la Educación Superior y Cooperación Internacional (PRIESCI), se considera uno de los más relevantes en la articulación de dinámicas de CSS. Dado que trabajó en el desarrollo de estrategias de integración con América del Sur, con el objetivo de promover la Internacionalización de la Educación Superior de acuerdo a los siguientes ejes de trabajo: integración, acreditación regional, reconocimiento de títulos vinculados a la acreditación, movilidad y asociación académica a nivel de posgrado. Los principales socios fueron: a nivel bilateral: Brasil; a nivel multilateral: MERCOSUR y UNASUR (Larrea \& Astur, 2011).

En el año 2013, el PRIESCI incorporó como instrumento de política la promoción de Redes del Núcleo de Estudios e Investigaciones en Educación Superior del Mercosur (NEIES), que privilegió los circuitos regionales de producción y difusión de conocimiento. En el caso de las redes regionales de producción y difusión de conocimiento, entendidas como dinámicas no hegemónicas que lograron un poder de agencia relativo a partir de: orientación de recursos, creación de espacios institucionales y generación de aprendizajes interinstitucionales, que contribuyeron a tensionar el proceso de internacionalización hegemónico (Oregioni \& Piñero, 2017).

No obstante, en la búsqueda de articular estrategias de políticas públicas en función de un proyecto nacional, en el año 2012 el Ministerio de Educación y el Ministerio de Ciencia, Tecnología e Innovación Productiva, comenzaron a debatir estrategias nacionales de formación de recursos humanos en el exterior en relación con áreas, campos y disciplinas estratégicas para el desarrollo nacional (MINCYT, 2012). En este 
sentido, se comenzaron a problematizar las dificultades que emergían hasta el momento, donde la vinculación internacional estaba dada a partir de estímulos exógenos, vinculados a la reproducción de la comunidad científica.

De esa forma, junto a la búsqueda de articulación interinstitucional, emergen cambios como la identificación de áreas de trabajo en función de un proyecto nacional, y, consecuentemente, la orientación de financiamiento en función de dos objetivos: 1) la vinculación territorial con Latinoamérica; y 2) los planes estratégicos de desarrollo nacional. Para ello, fueron convocados actores del sistema productivo, representantes de los Ministerios de Economía, Industria y Agricultura, de Fundaciones y Organismos Estatales con el fin de exponer sus experiencias y necesidades, que servirán de insumo para la formulación de proyectos en vinculación con las universidades.

Es decir que, desde el Ministerio de Educación, mediante la Secretaría de Políticas Universitarias, se llevaron adelante iniciativas de cooperación e internacionalización acorde al proyecto político nacional, consecuentemente, se buscó articular iniciativas con el MINCYT.

\section{Ministerio de Ciencia, Tecnología e Innovación Productiva}

Desde el Ministerio en Ciencia, Tecnología e Innovación Productiva (MINCYT), creado en el año 2007, la presencia internacional se plantea como un punto clave. Para tal fin, se creó la Dirección Nacional de Relaciones Internacionales, que, en el periodo de estudio, estuvo a cargo de la Lic. Águeda Menvielle, ${ }^{9}$ quien venía trabajando desde el año 1998 en el ámbito de la SECYT.

\footnotetext{
9 Quien sostiene la importancia que adquiere orientar la cooperación hacia el ámbito regional, en ese sentido, lo identifica como uno de los objetivos que se propone su gestión, haciendo referencia a la potencialidad que adquieren los posicionamientos conjuntos en las negociaciones internacionales del sector (Véase https://www.youtube.com/ watch? $=\mathrm{NZrNghFoOl)}$.
} 


\section{0 / Soledad Oregioni}

Los objetivos que plantea dicha dirección, consisten en complementar, fortalecer e integrar las capacidades de investigación y desarrollo del ámbito local con las del exterior, impulsando la realización de proyectos conjuntos de investigación, capacitaciones, intercambios de expertos y transferencias de resultados al sector productivo argentino. La dirección se ocupa de: a) supervisar los acuerdos internacionales; b) implementar proyectos conjuntos; c) diseñar instrumentos de vinculación y coordinar las relaciones de cooperación internacional de los distintos organismos del Sistema Nacional de Ciencia, Tecnología e Innovación y con el Ministerio de Relaciones Exteriores, Comercio Internacional y Culto; d) atender los temas de cooperación científica y tecnológica en el proceso de integración de los países del MERCOSUR; y e) articular la vinculación entre investigadores argentinos residentes en el país y en el extranjero.

Dentro de las competencias del MINCYT, con llegada a las universidades, se encuentra la Agencia Nacional de Promoción Científica y Tecnológica ${ }^{10}$ (ANPCYT), que promovió la cooperación a través del FONCYT (Bertranou, 2012), y el Consejo Nacional de Investigaciones Científicas y Técnicas (CONICET), que tiene una Agencia de Cooperación Internacional por medio de la cual se desarrollan vínculos y convenios internacionales y cuenta con diferentes instrumentos cofinanciados, como: los Programas de Intercambio Científico; Programa de Visitas Científicas, que permite a los investigadores científicos realizar visitas cortas en el extranjero, donde se cofinancia el viaje y la estadía. Las modalidades de cooperación consisten en: Grupos de investigación Internacionales (GII); Laboratorios Internacionales Asociados (LIA); Programas de Cooperación Internacional. En todos los casos, la Cooperación Sur-Sur y con la Región Latinoamericana, es la menos frecuente.

Sin embargo, desde el año 2011, CONICET busca promover los vínculos de cooperación con los países de la Región a partir del programa de becas doctorales y posdoctorales para estudiantes latinoamericanos,

\footnotetext{
10 Creada en el año 1996, desde 2007 se integra al MINCYT.
} 
destinado a estudiantes extranjeros, que se encuentran residiendo en un país latinoamericano en el momento de la beca. Tal como consta en la Res. 3224/11, las solicitudes deberán contener la propuesta de un director y lugar de trabajo en la Argentina y un codirector en el país de origen y, luego de completar su formación doctoral o, eventualmente, postdoctoral, los becarios tendrán la obligación de regresar a su país de origen y permanecer allí por un tiempo no menor al que ha usufructuado su beca.

Además, se identificaron distintos programas gestionados desde el MINCYT, destinados a ampliar los canales de colaboración con países de la Región, dando continuidad a las políticas desarrolladas por la Secretaria de Ciencia y Tecnología (SECYT), por ejemplo: el Programa de apoyo al desarrollo de la biotecnología en el MERCOSUR (BIOTECH), que desde el 2005 gestiona la plataforma Regional de biotecnologías BIOTECHSUR, o un ámbito de gestión como es la Reunión Especializada en Ciencia y Tecnología del MERCOSUR (RECYT), creada en la década del noventa. Sin embargo, en ambos casos resulta fundamental la mediación de Programas de cooperación extra regionales, provenientes fundamentalmente de la Unión Europea (Oregioni, 2012; Kern, 2014). También participó del Consejo Sudamericano de Ciencia, Tecnología e Innovación (COSUCTI), de la UNASUR.

Si bien en el caso del MINCYT, se identificó un discurso explícito que priorizaba la Región latinoamericana en correlación con la política exterior, en la práctica concreta, se continuó priorizando los vínculos de cooperación con los países del Norte, fundamentalmente con la Unión Europea, que incluso fue la mediadora de las relaciones de cooperación Sur-Sur de carácter multilateral. Esto se materializa en la cantidad de convenios firmados y activos donde la vinculación Norte-Sur fue prioritaria (Demarchi, 2018).

Asimismo, el programa de becas que promueve el CONICET, para estudiantes latinoamericanos, permite visibilizar una arista política que se va abriendo desde el organismo, que da cuenta del objetivo de generar capacidades en la región y fortalecer los vínculos de cooperación entre investigadores latinoamericanos. 


\section{Orientación de la cooperación ¿Qué lugar tiene la política?}

La CSS puede presentarse en un sentido restringido o en un sentido amplio (Milesi, 2015). En el periodo de estudio, se presentó en ambas modalidades. En un sentido restringido, vinculada a la cooperación técnica, podemos identificar la dinámica de cooperación que se promueve desde el FOAR. En un sentido amplio, a partir de la construcción de espacios políticos, ideológicos, con base en lazos de cooperación solidaria, que se manifiestan en la construcción de alianzas en organismos multilaterales, como se observa en la Conferencia Regional en Educación Superior del 2008 (Conferencia Regional, 2009), y la consolidación del Sector Educativo del Mercosur (Plan de acción, 2011), que se identifican con el trabajo que se realizó desde la Secretaria de Políticas Universitarias dependiente del Ministerio de Educación Argentino.

Ahora bien, los casos de estudio llevan a indagar en las posibilidades y limitaciones de los instrumentos políticos a la hora de orientar la cooperación en ciencia, tecnología y universidad, con el fin de conducir el proceso de internacionalización universitaria desde una perspectiva endógena, es decir, de acuerdo a los lineamientos de la política exterior. Consecuentemente, puntualizamos en la relación agencia-estructura, como co-construcciones dialécticas, que se generan desde una perspectiva situada.

En este sentido, siguiendo la política de cooperación llevada adelante por el FOAR y por la SPU, se puede argumentar que en el periodo 20032015 el Estado, cuya política exterior tiene objetivos autonomistas, se correspondió con la promoción de instrumentos que favorecen un modelo de internacionalización universitaria endógena. Sin embargo, en el caso de la política de cooperación en ciencia y tecnología que llevó adelante el MINCYT, si bien se desarrollaron instrumentos de CSS, se continuó priorizando los programas de cooperación Norte-Sur (Demarchi, 2018), incluso en ámbitos de cooperación de carácter multilateral y regional, como la RECYT o BIOTECH, estuvieron mediados por dinámicas de cooperación extra regionales (Oregioni, 2012; Kern, 2014). Es 
decir, que la cooperación en ciencia y tecnología se tensionó hacia una orientación dependentista, influenciada por patrones históricos del desarrollo de la ciencia en los países latinoamericanos, entre ellos la incidencia indirecta que ejercen los parámetros de evaluación de la ciencia (Oregioni, 2014) y su carácter colonial (Beigel, 2013). Dando cuenta de la orientación exógena de la internacionalización, guiada por los parámetros de internacionalización hegemónica (Oregioni, 2017) que, siguiendo a Robert Cox (2013-2014), forman parte de condicionantes estructurales a partir del sistema de creencias respecto a que es considerado conocimiento de "excelencia" y a los recursos materiales e institucionales que sostienen su reproducción.

Consecuentemente, al contemplar un abordaje holístico de las políticas de cooperación desarrolladas desde diferentes dependencias del Estado, como política pública y en relación a la política exterior, podremos observar que: entre 2007-2015 se buscó tejer alianzas estratégicas en función de un proyecto nacional y regional de carácter autonomista que, en algunos casos, entró en tensión con elementos estructurales e incentivos globales funcionales, a dinámicas hegemónicas, que históricamente guiaron dichas prácticas de internacionalización, reproduciendo dinámicas dependentistas que no se correlacionaban con la orientación de la política exterior del periodo. Esto muestra diferencias en la orientación de las políticas de cooperación por parte de las distintas instituciones nacionales que se vinculan con las universidades.

Además, lo expuesto, visibiliza la complejidad que adquiere la dinámica agencia-estructura, ${ }^{11}$ donde el poder de agencia, o margen de maniobra, está dado por la capacidad del Estado en definir sus propias políticas y agendas de investigación (a partir de ideas, intereses, instituciones, recursos), mientras que esta se encuentra condicionada por estructuras históricas, que se expresa a partir de ciertos elementos arraigados en el imaginario social, moldeando los pensamientos y acciones de los tomadores de decisiones que están al frente de las instituciones.

\footnotetext{
11 Para profundizar en el debate sobre agencia estructura en las Relaciones Internacionales véase Caballero (2018).
} 
De esta forma, se considera central entender la dinámica de co-construcción agencia-estructura, como un proceso bidireccional, en la definición de las políticas de cooperación como elementos para promover la internacionalización universitaria. Teniendo en cuenta que, desde una perspectiva holística, las políticas de cooperación se ven influenciadas por el proceso de internacionalización universitaria, a la vez que inciden en su orientación. Esto da lugar a tensiones entre diferentes formas e instrumentos de proyectar la internacionalización de las universidades, que contribuyen a legitimar o a cuestionar el proceso de internacionalización hegemónico.

De este modo, de acuerdo con Caballero "podemos afirmar la posibilidad de cambio en las estructuras, motivados por decisiones adoptadas por los agentes" (2018, p. 46), en contextos donde la correlación de fuerza es favorable, como fue el caso del regionalismo poshegemónico.

\section{Reflexiones finales}

Retomando la hipótesis de trabajo, en el periodo 2007-2015 el Estado argentino, de acuerdo con los ideales autonomistas que guiaron su política exterior, generó políticas de CSs que contribuyeron a orientar la internacionalización universitaria. De esta forma influyó en tensionar el proceso de internacionalización hegemónico, fundamentalmente en el plano de las ideas, avanzando en la consolidación de dinámicas institucionales y movilización de recursos.

No obstante, se presentaron elementos que permiten problematizar dicha articulación en torno al proyecto nacional y Regional. Dado que la política de CSs, que llevó adelante Argentina en relación con las universidades, no adquirió la misma intensidad en las diferentes dependencias estatales. Tal como se analiza en los apartados precedentes, en organismos como el MINCYT, si bien a nivel discursivo se alineó a los postulados de política exterior, en términos concretos la cooperación con los países del Norte continúo siendo prioritaria, mientras que desde la SPU y desde el FOAR, la región sudamericana 
ocupó un lugar central tanto en términos discursivos como en elementos de promoción.

Lo anteriormente expuesto, evidencia que es posible contribuir a orientar el proceso de internacionalización universitaria en correlación con una perspectiva autónoma de política exterior, mediante políticas de CSS, en contraposición con el proyecto de internacionalización hegemónico en términos de la globalización neoliberal y neocolonial (que sigue la lógica del mercado, orientada por las agendas que fijan los Organismos Financieros Internacionales, llevando a las universidades a competir por posicionarse en ranking internacionales, impone el inglés como lengua franca y se aspira a un modelo de universidad de "clase mundial").

Es decir que, en las fisuras de un proceso de internacionalización hegemónico inconcluso, emergen dinámicas de css que buscan orientarlo hacia la integración regional, retomando los ideales latinoamericanos de autonomía y desarrollo endógeno. Para ello se movilizaron recursos desde distintas dependencias del Estado en articulación con las universidades. Ahora bien, para que se materialice en dinámicas de cooperación, es necesario que la universidad y sus investigadores comprendan la importancia de promover dinámicas de internacionalización alternativas, con base en lazos de CSs. Entendiendo al Sur, no solamente desde una perspectiva geográfica, sino fundamentalmente desde una perspectiva cultural y política.

Para finalizar, el trabajo deja planteada la necesidad de discutir la coconstrucción agencia-estructura en la consolidación de procesos históricos, como es el caso del proceso de internacionalización universitaria. Esto resulta fundamental para los países latinoamericanos, como Argentina, que en diferentes ocasiones se encontraron presos de trampas estructurales, que llevaron a justificar su destino de subordinación.

En síntesis, el trabajo destacó la incidencia directa de la política de cooperación, vinculada a la política exterior, en generar instrumentos para orientación del proceso de internacionalización, que permitan: socializar nuevas de ideas, movilizar recursos, y generar dinámicas 
institucionales de CSS, contribuyendo a tensionar el proceso de internacionalización hegemónico, arraigado en dinámicas estructurales.

\section{Referencias bibliográficas}

Alonso, J. A., Aguirre, P., \& Santander, G. (2011). La cooperación Internacional Española en América Latina: un análisis de dos experiencias de interés [Documento de trabajo $\mathrm{N}^{\circ}$. 51]. Fundación Carolina; Instituto Complutense de Estudios Internacionales, ICEI. https://www.fundacioncarolina.es/wp-content/uploads/2014/08/DT51.pdf

Altbach, P. (2004). Globalization and the university: Myths and realities in an unequal World. Tertiary Education and Manangement, (10), 3-25. https:// link.springer.com/article/10.1023/B:TEAM.0000012239.55136.4b

Beigel, F. (2013, mayo-junio). Centros y periferias en la circulación internacional del conocimiento. Nueva Sociedad, 245, 110-123. https://static. nuso.org/media/articles/downloads/3944_1.pdf

Beigel, F., \& Sabea, H. (Comps.). (2014). Dependencia Académica y Profesionalización en el Sur. Perspectivas desde la Periferia [Colección Encuentros, 4]. EDIUNC; SEPHIS.

Bertranou, A (2012). La Formación de Recursos Humanos y la Promoción de Cooperación Internacional desde la Agencia. En MINCYT (Ed.), Hacia un mejor aprovechamiento de la cooperación internacional para el fortalecimiento del sistema nacional en ciencia, tecnología e innovación (pp. 42-44). MINCYT. http://raices.mincyt.gov.ar/documentos/Hacia\%20un\%20 mejor\%20aprovechamiento.pdf

Caballero, S. (2018). El debate Agencia-Estructura en las Relaciones Internacionales: Capacidad de agencia y limitaciones estructurales. En F. Verdes-Montnegro \& N. Comini (Coords.), Otras miradas y otras voces. Visiones Críticas de las relaciones internacionales. (pp. 29-47). UNTREF.

Cetto, A., \& Vessuri, H. (1998). América Latina y el Caribe. En Informe Mundial sobre Ciencia (pp. 55-75). UNESCO.

Colacrai, M., Kern, A., Vallone, M., Pattacini, V., \& Weisstaub, L. (2009). Escenarios y desafios de la cooperación Sur-Sur a 30 años de la declaración de Buenos Aires. [Documento de Trabajo $\mathrm{N}^{\circ} 1$ ]. Centro de Investigación en Cooperación Internacional y Desarrollo de la Escuela de Humanidades de la Universidad Nacional de San Martín, Argentina. 
Colin, M.-O. (2001, primavera). La cooperación académica y científica como dimensión de la política exterior. Política y Cultura, (15). https:// www.redalyc.org/articulo.oa?id $=26701503$

Colombo, S. (2011). La inserción internacional de Argentina durante la presidencia de Néstor Kirchner. Un cambio de época. UNCPBA

Conferencia Regional de la Educación Superior en América Latina y el Caribe (CRES). Declaraciones y plan de acción. (2009). Perfiles educativos, 31(125), 90-108. http://www.scielo.org. $\mathrm{mx} /$ scielo.php? script $=$ sci_ arttext\&pid $=$ S0185-26982009000300007\&lng=es\&tlng=es.

Corder, S. Costa, M. C., M., Gomes, E., Velho, P. E. (2002). Mercosur: Cooperación en ciencia y tecnología, Nueva antropología, 18(60), 9-28. https://www.redalyc.org/pdf/159/15906002.pdf

Cox, R. (2013, octubre - 2014, enero). Fuerzas sociales, estados y órdenes mundiales: Más allá de la teoría de las Relaciones Internacionales. Revista Relaciones Internacionales, 24, 129-162. https:/ / revistas.uam.es/ index.php/relacionesinternacionales/ article/view/5195/5641

De Filippo, D., Morillo, F., \& Fernández, M. T. (2008). Indicadores de colaboración científica del CSiC con Latinoamérica en bases de datos internacionales. Revista Española de Documentación Científica, 31(1), 66-84. http:/ / digital.csic.es/bitstream/10261/11663/1/425.pdf

De Sousa-Santos, B. (2008). El de la universidad en la construcción de una globalización alternativa. La Educación Superior en el mundo, 3(9), 169171. https://upcommons.upc.edu/handle/2099/7981.

De Sousa-Santos, B. (2017) Justicia entre saberes. Epistemologías del sur contra el Epistemicidio. Morata.

Demarchi, P. (2018, enero-junio). La cooperación internacional en ciencia y tecnología argentina: Análisis de la relación política explícita política implícita en el período 2007-2013. Revista Integración y Cooperación Internacional, 26. https:/ / rephip.unr.edu.ar/handle/2133/13580

Dussel, E. (2000). Europa, modernidad y eurocentrismo. En La colonialidad del saber: eurocentrismo y ciencias sociales. Perspectivas Latinoamericanas (pp. 24-33). CLACSO. http://bibliotecavirtual.clacso.org.ar/clacso/sursur/20100708040738/4_dussel.pdf

Elorduy, P. (2018, 19 de mayo). Boaventura de Sousa: "La tragedia de nuestro tiempo es que la dominación está unida y la resistencia está fragmentada". El Salto. https://www.elsaltodiario.com/pensamiento/ entrevista-boaventura-sousa-tragedia-nuestro-tiempo-dominacion- 
unida-resistencia-fragmentada\#: : :text $=$ Pensamiento-,Boaventura $\% 20$ de $\% 20$ Sousa $\% 3 \mathrm{~A} \% 20 \%$ E2 $\% 80 \% 9$ CLa $\% 20$ tragedia $\% 20$ de $\% 20$ nuestro $\% 20$ tiempo $\% 20$ es $\% 20$ que,otro $\% 20$ mundo $\% 20$ distinto $\% 20$ al $\% 20$ capitalista.

Fernández, C. (2019). Cristina Fernández de Kirchner. Una politica exterior soberana. Colihue.

Fernández, M., Gómez, I., \& Sebastián, J. (1998). La cooperación científica de los países de América Latina a través de indicadores bibliométricos. Revista Interciencia, 23(6), 328-336.

Funes, M. (2014). Internacionalización de la Educación Superior en Argentina. En G. Tangelson (Comp.), Desde el sur. Miradas de la internacionalización (pp. 39-52). Universidad Nacional de Lanús: Argentina. http:// obiret-iesalc.udg.mx/sites/default/files/publicaciones/16._desde-elsur-miradas-sobre-la-internacionalizacion.pdf

García-Guadilla, C. (2010). Heterogeneidad y concentración en las dinámicas geopolíticas del conocimiento académico. Reflexiones y preguntas para el caso de América Latina. En M. Mollis (Ed.), Politicas de posgrado y conocimiento público en América Latina y el Caribe: desafíos y perspectivas (pp. 135-164). Consejo Latinoamericano de Ciencias Sociales, Buenos Aires, Argentina.

Grugel, J., \& Riggirozzi, P. (2012). Post-neoliberalism in Latin America: Rebuilding and Reclaiming the State after. Crisis. Development and change, 43(1), 1-21.https://eprints.soton.ac.uk/300440/1/Dev_and_Change_2012.pdf

Gusmão, R. (2000). La implicación de los países latinoamericanos en los Programas Europeos de Cooperación Científica y Tecnológica con terceros países. Revista Redes, 7(16) 131-163. https://www.redalyc.org/ pdf/907/90701605.pdf

Hurtado, D. (2012). La colaboración científica en dos ejes de cooperación clave Sur-Sur (s-S) y Norte-Sur (N-S). En MINCYT (Ed.), Hacia un mejor aprovechamiento de la cooperación internacional para el fortalecimiento del sistema nacional en ciencia, tecnología e innovación (pp. 24-27). http:/ / raices.mincyt. govar/documentos/Hacia\%20un\%20mejor\%20aprovechamiento.pdf Kern, A. \& Weisstaub, L. (2019). La cooperación Sur-Sur de Argentina. En T. Ojeda-Medina \& E. Muñoz (Comps.), La Cooperación Sur-Sur en América Latina y el Caribe. Balance de una década (2008-2018) (pp. 41-52). CLACSO. 
https://www.jstor.org/stable/j.ctvt6rmgq.5?seq=1\#metadata_info_tab_contents

Kern, A. (2008). Relaciones entre ciencia, tecnología y política en procesos de cooperación internacional. Análisis de un caso de cooperación entre Argentina y Alemania en el campo de las Tecnologías Informáticas [Tesis doctoral] Facultad Latinoamericana de Ciencias Sociales. Sede Académica Argentina.

Kern, A. (2014). La agenda científica y tecnológica en los regionalismos de América Latina. Conferencia Internacional Conjunta FLACSO-ISA. http:/ / web.isanet.org/Web/Conferences/FLACSOISA\%20BuenosAires \%20 2014/Archive/9705df48-1e10-4e32-95fe-f46fc48f5992.pdf

Kreimer, P. (2006). ¿Dependientes o integrados? La ciencia latinoamericana y la división internacional del trabajo. Nómadas, 24, 199-212. https:// www.redalyc.org/pdf/1051/105116598017.pdf

Kreimer, P. (2014). ¿Ciudadanos del Mundo o productores de conocimiento útil? Esa es la cuestión. En H. Sabea \& F. Beigel (Eds.), Dependencia Académica y Profesionalización en el Sur. Perspectivas desde la Periferia (pp. 151-166) [Colección Encuentros, 4]. EDIUNC; SEPHIS.

Kreimer, P., \& Levin, L. (2011). Mapping trends and patterns in S\&T Cooperation between the European union and Latin American countries based on FP6 and FP7 projects. En J. Gaillard \& R. Arvanitis (Eds.), Mapping and understanding science and technology collaboration between Europe and Latin America (pp. 79-104). L'Institut de Recherche pour le Développement.

Larrea, M., \& Astur, A. (2011). Políticas de internacionalización de la educación superior y cooperación internacional universitaria [Archivo pdf]. http://pep.unc.edu.ar/wp-content/uploads/sites $/ 46 / 2017 / 02 / \mathrm{Pol}_{0} \% \mathrm{C} 3 \%$ ADticas-de-internacionalizaci $\% \mathrm{C} 3 \% \mathrm{~B} 3 \mathrm{n}$ de-la-Eduaci $\%$ C3\%B3n-Superior-Larrea-M-y-Astur-A.pdf

Leal, F., \& Oregioni, M. (2019, febrero). Aportes para analizar la internacionalización de la educación superior desde Latinoamérica: un enfoque crítico, reflexivo y decolonial. Revista Internacional de Educação Superior, 5,1-19. Doi 10.20396/riesup.v5i0.8653635

Lechini, G. (2009, octubre) La cooperación Sur-Sur y la búsqueda de autonomía en América Latina: ¿Mito o realidad? Relaciones Internacionales, 12, 55-81. https:/ / revistas.uam.es/index.php/relacionesinternacionales / article/view/4980/5443 
Losego, P., \& Arvanitis, R. (2008). La ciencia en los países no hegemónicos. Revue d'anthropologie des connaissances, 2(3), 351-359. https:/ /www.cairn. info/revue-anthropologie-des-connaissances-2008-3-page-351.htm

Malacalza, B. (2014, julio-diciembre). La política de cooperación al desarrollo como dimensión de la política exterior desde la Teoría de las Relaciones Internacionales. Mural Internacional, 5(2), 163-176. https:/ / www.e-publicacoes.uerj.br/index.php/muralinternacional/article/ viewFile/10771/12128

Marí, M., Estébanez, M. E., \& Suárez, D. (2001, junio). La Cooperación En Ciencia y Tecnología de Argentina con los Países del Mercosur. Revista Redes, 8(17), 59-82. https://www.redalyc.org/pdf/907/90781702.pdf

MINCYT. (2012). Hacia una Argentina innovadora. Plan Nacional en Ciencia, Tecnología e Innovación (2012-2015). https:/ /issuu.com/asap1/docs/ pncti2012-2015

Milesi, C. (2015). Cooperación Sur-Sur. El Caso de Argentina [Documento de trabajo]. http://ceciliamilesi.com/wp-content/uploads/2015/03/ CSS-Argentina-Milesi.pdf

Morales-López, H. (2012) Construyendo lazos de solidaridad, dignidady soberanía. Cooperación Sur-Sur. Mugarik Gabe. https://www.mugarikgabe.org/wpcontent/uploads/2015/05/Libro-Cooperaci\%C3\%B3n-Sur-sur.pdf

Morasso, C. (2016, enero-junio). La orientación autonomista de la política exterior Argentina. Cuadernos de Política Exterior Argentina (Nueva Época), 123, 3-22. https://core.ac.uk/download/pdf/84263774.pdf

Oregioni, M. (2012). Trayectoria político-institucional de la cooperación en ciencia y tecnología en el Mercosur. El caso de la reunión Especializada en Ciencia y Tecnología (RECYT) (1992 -2008) [Tesis de Maestría]. Universidad Nacional de Quilmes, Argentina.

Oregioni, M. (2013). La Universidad como actor de la cooperación Sur-Sur. El caso de la Universidad Nacional de la Plata en la Asociación de Universidades Grupo Montevideo (AUGM). Revista Integración y Conocimiento, (2), 53-67. https://revistas.unc.edu.ar/index.php/integracionyconocimiento/article/view/5893

Oregioni, M. (2014). Dinámica de Internacionalización de la Investigación en la Universidad Nacional de La Plata (2005-2012) [Tesis Doctoral]. Universidad Nacional de Quilmes, Argentina. https://ridaa. unq.edu.ar/bitstream/handle/20.500.11807/120/OREGIONI. pdf ? sequence $=1 \&$ is Allowed $=\mathrm{y}$ 
Oregioni, M. (2015, agosto 12-14) ¿De qué hablamos cuando hablamos de internacionalización universitaria? [Pre-ALAS 2015]. En Desafíosy Dilemas de la Universidad y La Ciencia En América Latina y El Caribe en el Siglo XXI. Facultad de Ciencias Sociales, Universidad de Buenos Aires. Oregioni, M. (2016). Aportes conceptuales sobre Internacionalización Universitaria. Una mirada desde América Latina. En M. Oregioni y F. Piñero (Comps.), Herramientas de Política y Gestión para la Internacionalización Universitaria. Tandil:Grafikat

Oregioni, M. (2017, julio-diciembre). Internacionalización de la investigación. Las unidades de investigación de la Universidad Nacional de La Plata. Revista https:// revistas.itm.edu.co/index.php/trilogia/article/ view/629

Oregioni, M., \& Abba J. (2012). Política de cooperación hacia América Latina en el marco de la cooperación Sur-Sur. El caso del FO-AR (2003-2010). En A. Piñero (Comp.), Ciencia y Tecnología en la argentina Contemporánea. Dimensiones para su Análisis (pp. 169-192). UNCPBA.

Oregioni, M., \& Daddario, M. (2016, noviembre). Internacionalización Universitaria y Politica Exterior en un contexto de Cambios. VIII Congreso de Relaciones Internacionales, Universidad Nacional de La Plata.

Oregioni, M., \& Piñero, F. (2009). Política Argentina de cooperación en ciencia y tecnología. Análisis de la incidencia de los programas marco de la Unión Europea en la definición de agendas. En A. VidalesCarmona, A. Figueroa-Delgado y G. Sánchez Daza (Eds.), La Ciencia y Tecnología en el Desarrollo. Una visión desde América Latina (pp. 53-65). http://biblioteca.clacso.edu.ar/Mexico/uacp-uaz/20100322012242/ CYTED.pdf

Oregioni, M., \& Piñero, F. (2017). Las redes como estrategia de internacionalización universitaria en el Mercosur. El caso de la RIESAL (20132017). Revista Integración y conocimiento. 1(6), 114-133. https:/ / revistas. unc.edu.ar/index.php/integracionyconocimiento/article/view/17121 Organización de Estados Iberoamericanos _OEA. (7 de junio de 2013). Entrevista Agueda Menvielle (Archivo de video]. https://www.youtube.com/watch?v=NZrNghFoOlI

Pereyra-Doval, G. (2014). Autonomía en la Política Exterior de Lula da Silva. En G. Lechini (Comp.), La cooperación Sur-Sur en las politicas exteriores de Argentina y Brasil en el siglo XXI (pp. 52-60). UNR https:// 
rephip.unr.edu.ar/bitstream/handle/2133/4468/LIBRO-CSSPEAB. pdf? seque nce $=3$

Pinhero, C., \& Martin, E. (2014). SEPHIS y la crítica a la Dependencia Académica en el Mundo Actual. En F. Beigel \& H. Sabea (Comp.) Dependencia Académica y Profesionalización en el Sur. Perspectivas desde la periferia (pp. 11-13) [Colección Encuentros, 4]. EDIUNC; SEPHIS.

Plan de acción del sector educativo del mercosur. (2011). Mercosur. http://edu.mercosur.int/es-ES/component/jdownloads/finish/7/414. html

Redrado, M. (2010). Sin reservas. Un límite al poder absoluto. Planeta. Riggirozzi, P., \& Tussie, D. (Eds.). 2012. The Rise of Post-hegemonic Regionalism: The Case of Latin America. Springer. https://link.springer. com/book/10.1007/978-94-007-2694-9

Romero, P. (2009). Hacia una nueva construcción discursiva de la autonomía. En G. Lechini, V. Klagsbrunn \& W. Gonçalves (Comps.), Argentina y Brasil: Venciendo preconceptos: Las variadas aristas de una concepción estratégica. Revan.

Russell, J. M., Ainsworth, Sh., Del Río, J. A., Narváez-Berthelemot, N., \& Cortés, H. D. (2007, abril-junio). Colaboración científica entre países de la región latinoamericana. Revista española de documentación científica, 30(2), 180-198. http://redc.revistas.csic.es/index.php/redc/article/ view $/ 378$

Sanahuja, J. A. (2010). La Construcción de una Región: Suramérica y el Regionalismo Posliberal. En M. Cienfuegos, \& J. A. Sanahuja (Eds.), Una región en construcción: Unasur y la integración de América del Sur (pp. 87-134). Fundación Cidob.

Sancho, R., Morillo, F., De Filippo, D., Gómez, I., \& Fernández, M. T. (2006). Indicadores de colaboración científica inter-centros en los países de América Latina. Revista Interciencia, 31(4), 284-292.

Sebastian J. (2004). Cooperación e Internacionalización de la Universidades. Biblos. Serbin, A., Martínez, L., \& Ramanzini-Jr, H. (2012). El regionalismo "postliberal" en América Latina y el Caribe: Nuevos actores, nuevos temas, nuevos desafíos. Anuario de la Integración Regional de América Latina y el Gran Caribe 2012. CRIES. http://www.cries.org/wp-content/ uploads/2013/03/anuario2012.pdf 
Simonoff, A. (2009). Regularidades de la política exterior de Néstor Kirchner. Revista CONfines, 5(10), 71-86. http://www.scielo.org.mx/pdf/ co nfines/v5n10/v5n10a6.pdf

Surasky, J. (2010). Argentina y la Cooperación Sur-Sur, En B. Ayllón \& J. Surasky (Comps.), La cooperación Sur-Sur en Latinoamérica. Utopía y Realidad (Capítulo 2). Instituto Universitario de Desarrollo y Cooperación, Universidad Complutense de Madrid. https://www.ucm.es/ data/cont/media/www/pag-82466/SERIE_35.pdf

Velho, L. (2000, agosto). Redes regionales de cooperación en CyT en el MERCOSUR. Revista Redes, 7(15), 112-130. https://www.redalyc.org/ pdf/907/90701504.pdf 